\title{
Monitorización de víctimas con manipuladores aéreos en operaciones de búsqueda y rescate
}

\author{
Francisco J. Ruiz-Ruiz, Juan M. Gandarias, Antonio J, Muñoz-Ramírez, Alfonso J. García-Cerezo, \\ Francisco Pastor-Martín y Jesús M. Gómez-de-Gabriel
}

Universidad de Málaga. Andalucía Tech. Dto. de Ingeniería de Sistemas y Automática. jmgandarias@uma.es

\begin{abstract}
Resumen
En este trabajo se presenta el primer dispositivo de monitorización de víctimas para su colocación automática con robots manipuladores aéreos. Se trata de un sistema sensorial distribuido para la evaluación de forma continua del estado de salud de víctimas de catástrofes. Se describen el sensor diseñado y el sistema de comunicaciones, así como la aplicación mediante la colocación del sensor basado en el uso de sistemas aéreos no tripulados (UAS) o robots manipuladores aéreos. El dispositivo de monitorización continua ofrece ventajas sobre el sistema de triage actual ya que permite obtener datos de la evolución de cada víctima. Recoge medidas de las constantes vitales de las víctimas, que son publicadas mediante protocolos de Internet de las Cosas (IoT) que permiten su procesado de forma remota. Además, posee métodos basados en aprendizaje profundo para la detección automática de la posición relativa de la muñeca del brazo de una persona con respecto al manipulador aéreo. Se han realizado experimentos preliminares de obtención de medidas y de colocación de sensores mediante una versión preliminar del sensor, cuyos resultados se incluyen.
\end{abstract}

Palabras clave: Robótica de rescate, Monitorización de víctimas, Internet de las Cosas, Impresión $3 \mathrm{D}$

\section{INTRODUCCIÓN}

En la actualidad, los accidentes con múltiples víctimas no suceden muy a menudo, pero cuando se dan es necesario atender a los heridos con la mayor brevedad posible y de forma organizada. El término triage hace referencia al proceso de clasificación de pacientes de urgencia según su gravedad, de forma que los equipos de búsqueda y rescate (SAR) puedan establecer un orden prioritario de atención a las víctimas según el nivel de urgencia. Este proceso que puede llegar a ser complejo [11]. En la actualidad, el personal sanitario realiza una evaluación rápida de cada una de las víctimas cada cierto tiempo. La prioridad asignada se muestra mediante una tarjeta de triage atada a la muñeca o el tobillo de la víctima [7].

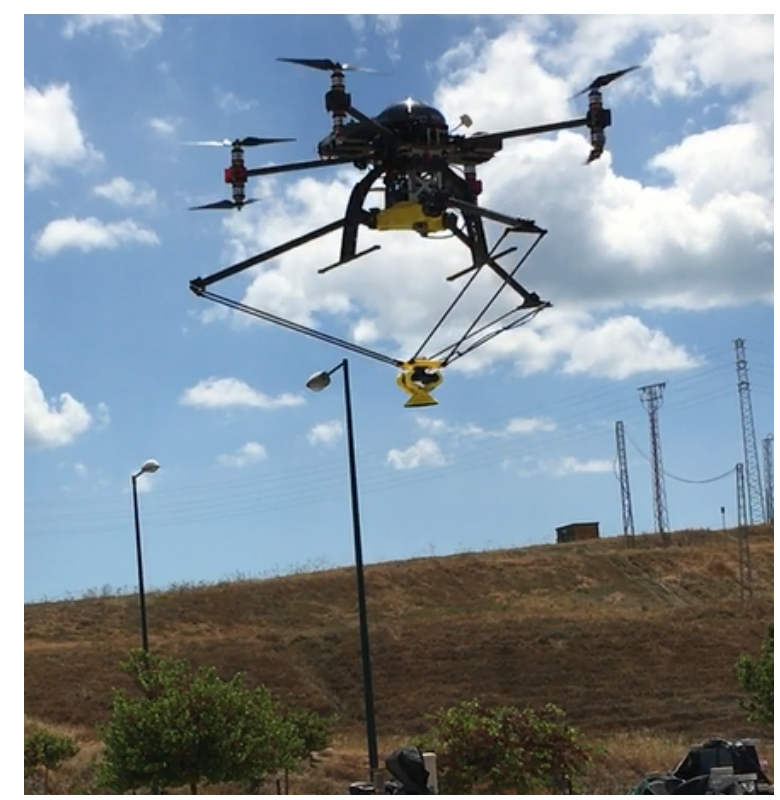

Figura 1: Manipulador aéreo con un sensor. El manipulador es un brazo de tres grados de libertad tipo delta adaptado a un octocóptero de Atyges.

Gracias al auge de los microcontroladores de bajo coste y las comunicaciones, es cada vez más factible el uso de redes sensoriales que permitan la monitorización de diversos parámetros, como son las constantes vitales de una persona [8]. Así pues, los avances en electrónica permiten la integración de sensores en espacios cada vez más reducidos, siendo posible colocarlos en objetos cotidianos [1].

De igual forma, los avances en la interacción robothumano hacen que sea la colocación de dispositivos sensoriales en personas mediante manipuladores robóticos pueda realizarse de forma segura [3]. Con el uso de robots para operaciones SAR, la rápida localización y la monitorización continua de víctimas es posible. En [2] se muestra cómo un sistema aéreo no tripulado (UAS) con múltiples sensores puede llegar a la víctima antes que los servicios de emergencias. En [6] se presenta la colocación de un sensor con comunicaciones inalámbricas en una víctima, de forma que se permite realizar ecografías de forma remota. Por otro lado, el uso de robots con dos canales de comunicaciones multimedia pa- 
ra la interacción con víctimas atrapadas ha sido previamente presentados en [9]. Sin embargo, estas soluciones mantienen al robot ocupado durante la monitorización. Por lo tanto, para escenarios de desastre masivos con múltiples víctimas, se requiere una solución distribuida que permita al robot atender a nuevas víctimas, mientras se monitoriza el estado de las víctimas previamente atendidas.

En este trabajo se presenta el desarrollo del primer dispositivo de monitorización del estado de salud de víctimas en operaciones SAR que permite su colocación automática mediante UAS. En la Figura 1 se muestra un manipulador aéreo portando un nodo sensor plano. Se describe el diseño de una pulsera sensorizada que recoge información acerca de las constantes vitales de una víctima, que es publicada en un servidor mediante protocolos de IoT. De esta forma, se provee un sistema de monitorización de víctimas en tiempo real. Este dispositivo de monitorización continua ofrece ventajas sobre los métodos de triage actuales ya que permite obtener datos de la evolución de cada víctima. Además se ha acoplado un manipulador robótico ligero tipo delta a un UAS. De forma que se puede realizar el acoplamiento de la pulsera a las víctimas de forma automática utilizando técnicas de aprendizaje profundo. Un trabajo previo de este grupo se presenta en $[5]$.

Este documento está organizado de la forma siguiente: En la sección 2 se describe el diseño del sensor remoto mediante su diseño mecánico y electrónico. A continuación, en la sección 3 se describe el sistema de comunicaciones implementado para obtener la información de los diferentes sensores remotos desplegados. En la sección 4 se describe el sistema de colocación automático de los sensores mediante el uso de manipuladores aéreos. Finalmente se incluyen resultados de experimentos y conclusiones.

\section{DISEÑO DEL SENSOR REMOTO}

El sistema debe recoger información acerca de las constantes vitales de una persona y enviar dicha información a un servidor web para su representación en tiempo real, empleando para ello comunicación inalámbrica. Las variables que se recogen son la frecuencia cardíaca (HR) y la saturación de oxígeno en sangre $\left(\mathrm{SPO}_{2}\right)$. Además, se ha integrado un sistema de detección del movimiento de la víctima (Mov). Además, desde la interfaz de usuario, se pueden enviar órdenes a la pulsera para que se active una señal luminosa mediante un anillo de Leds, y una señal sonora mediante un zumbador. Debido a que las constantes vitales no presentan, por lo general, cambios bruscos en periodos cortos de tiempo, se permite una latencia máxima entre envío de mensajes de 2 segundos. En la figura 2 se presenta un esquema funcional del sistema sensorial remoto.

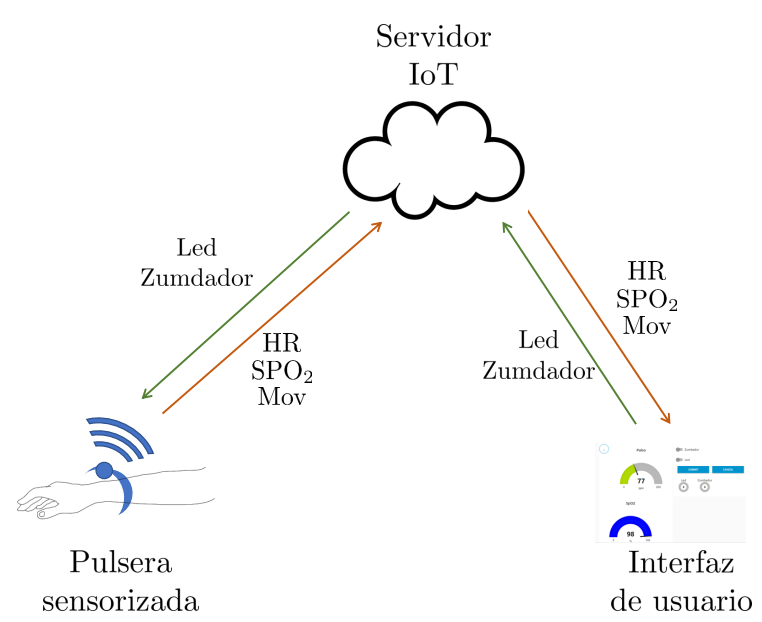

Figura 2: Esquema funcional del sistema sensorial remoto.

Una vez que el sensor es instalado, éste transmite información del estado de la víctima al servidor web. Desde la interfaz de usuario del mismo servidor, el personal del equipo de rescate puede monitorizar las constantes vitales de la víctima y enviar órdenes al nodo mediante IoT.

\subsection{Sistema sensorial}

Para la detección del movimiento de la persona se ha hecho uso de un acelerómetro, (módulo MPU92/60). Éste incorpora al MPU 9250 de Invesense, que posee un procesador digital de movimiento (DMP). Para la detección de la frecuencia cardíaca y la saturación de oxígeno en sangre, se ha usado un pulsioxímetro de reflexión, el MAX30102 de Maxim Integrated.

Por otro lado, se ha añadido un anillo de diodos LED RGB de NeoPixel que indica la prioridad de atención de la víctima. Además, sirve de señal indicativa para facilitar la localización de la víctima a los equipos de rescate. Asimismo, se ha hecho uso de un zumbador piezoeléctrico como sistema de actuación acústica, que también permite localizar a la víctima. Como elemento de control del sistema sensorial se ha empleado un módulo basado en el microcontrolador ESP32 de Espressif Systems, el módulo Wi-Fi LoRa 32 de Heltec Automation. En la Figura 3 se muestra el esquema de conexionado de los elementos del sistema.

\subsection{Diseño mecánico}

El sistema se integra en una pulsera que se acopla al brazo de la víctima. De esta forma podrá ser 


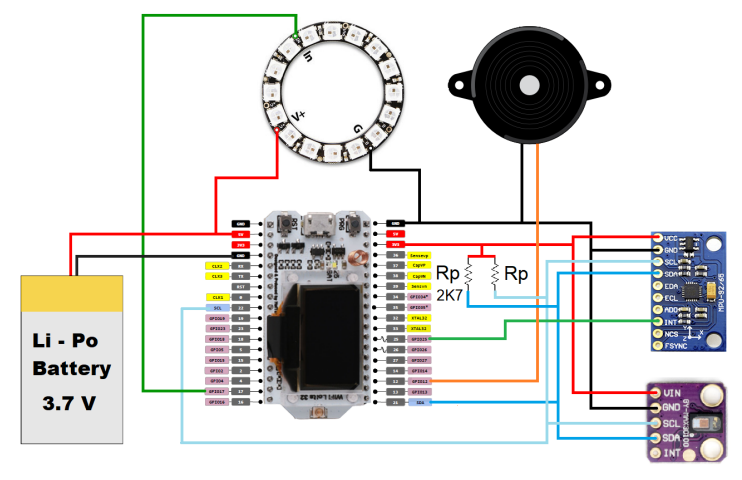

Figura 3: Esquema de conexionado de los distintos módulos del sistema electrónico del sistema sensorial. Está compuesto por un módulo de procesamiento Espressif ESP32 con un chip de radio LORA Semtech SX1276 y display OLED, además de una batería LiPO y los módulos de sensores MAX30102 y MPU-92/65. Asimismo el sistema cuenta con indicadores visuales anillo de LED WS2811 y zumbador.

colocada tanto por una persona como por un robot manipulador (terrestre o aéreo). El mecanismo de la pulsera está pendiente de ser patentado, por lo que no se detallan en esta sección las características del mismo. Para la construcción del prototipo se ha usado fabricación aditiva mediante una impresora 3D de tipo FDM (Modelado por Deposición Fundida) y material PLA (polylactic acid). En la imagen de la figura 4 se presenta el prototipo final de la pulsera impresa en 3D.

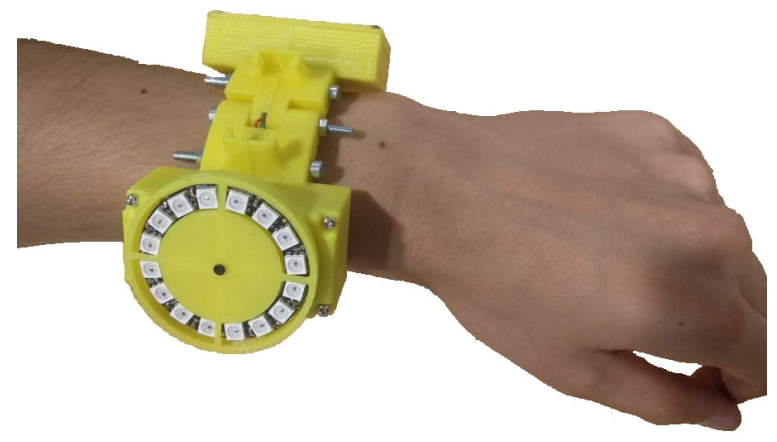

Figura 4: Fotografía de la pulsera impresa en 3D, colocada en el brazo de una persona y comunicándose mediante protocolo MQTT.

\section{COMUNICACIONES}

\subsection{Estructura del sistema distribuido}

Para el desarrollo de los experimentos se ha alojado un servidor en una placa Raspberry Pi 3. Ésta puede ser fácilmente transportada por un robot, tanto aéreo como terrestre. Este robot, en este caso, debe transportar, además, un router que proporcione conexión Wi-Fi a todos los sensores que se encuentren distribuidos a lo largo de la zona de catástrofe.

Por otro lado, desde la estación de control en tierra (GCS - Ground Control Station), el personal sanitario y los sistemas de planificación estratégica de los equipos de rescate puede conectarse a la red y acceder, de forma remota, al estado de las víctimas. Lo que les permite realizar un plan de evacuación y hospitalización de las víctimas en base a la urgencia de la atención.

En la Figura 5 se muestra un esquema del sistema completo. Las flechas que unen los distintos elementos representan el intercambio de información entre el servidor y los clientes mediante el protocolo MQTT.

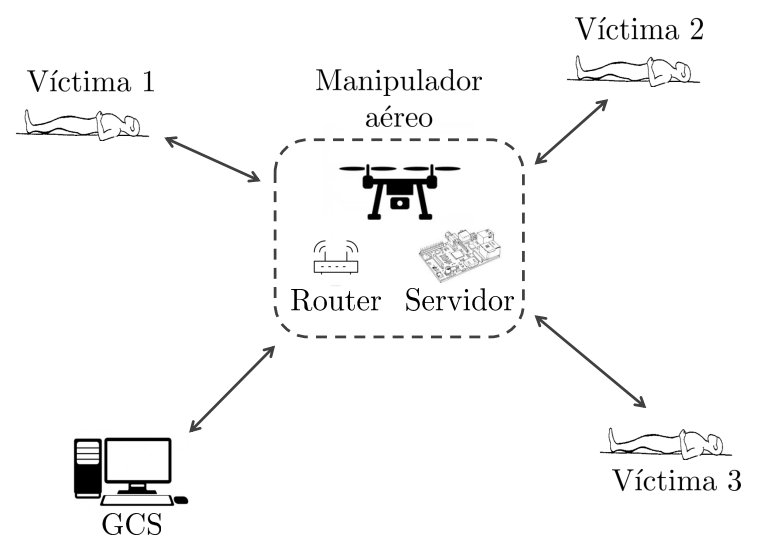

Figura 5: Esquema de funcionamiento del sistema distribuido.

\subsection{Desarrollo de la interfaz con Node-Red}

Para la programación del servidor se ha usado Node-Red. En la Figura 6 se pueden observar los flujos de recepción, a), y envío de mensajes, b). El flujo de recepción descompone el mensaje recibido y muestra los datos que este contiene. Cuando se altera el estado de los actuadores se envía un mensaje al nodo y se actualiza el estado de los mismos en la interfaz de usuario. Para recibir y enviar los mensajes MQTT es necesario el uso de un broker. Para este trabajo se ha empleado el broker MQTT Mosquitto. 


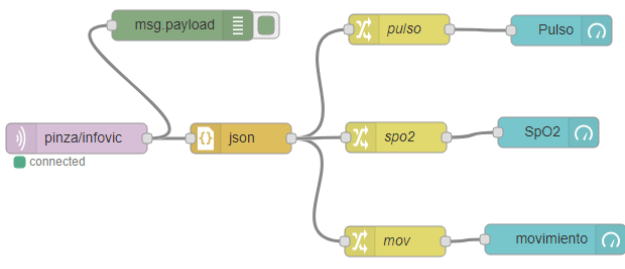

a)

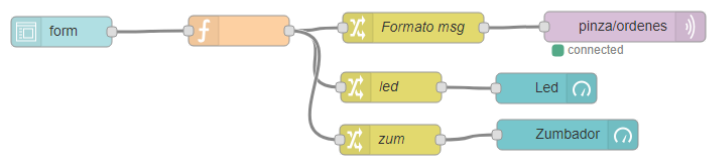

b)

Figura 6: Flujo de recepción, a), y envío, b), de mensajes generados en Node-Red.

En la Figura 7 se muestra la interfaz de usuario programada mediante la librería Dashboard. Dicha interfaz contiene los elementos necesarios para la monitorización del movimiento, frecuencia cardíaca (en pulsaciones por minuto, ppm) y saturación de oxígeno de la víctima (en tanto por ciento), así como para el control sobre los actuadores y la visualización de su estado.

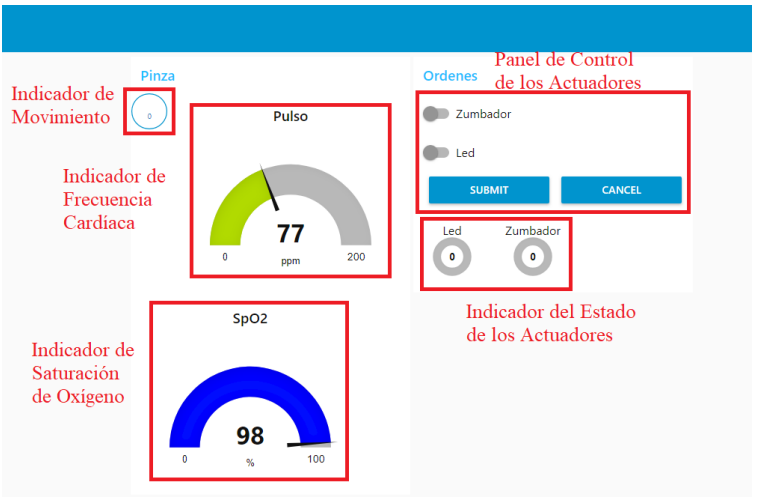

Figura 7: Interfaz de usuario. En la parte izquierda se representan los valores de la frecuencia cardíaca, la saturación de oxígeno y la detección de movimiento. En la parte derecha se pueden activar de forma remota el anillo de LEDs y el zumbador.

\subsection{Sistema de triage}

Asimismo, se ha implementado un sistema de clasificación de la urgencia de atención de las víctimas. Para ello se ha tenido en cuenta, de acuerdo con [4], que los niveles normales de pulso en un adulto sano están comprendidos entre 40 - 120 ppm. Por otro lado, el porcentaje mínimo de saturación de oxígeno en una persona adulta se ha establecido en un $95 \%$.El algoritmo de triage implementado
Tabla 1: Clasificación en estados de triage.

\begin{tabular}{lll}
\hline HR & $\mathrm{SpO}_{2}$ & Prioridad \\
\hline \hline $\mathrm{HR}<10$ & $\mathrm{SpO}_{2}<70$ & Negro \\
\hline $\begin{array}{l}\mathrm{HR}>150 \\
\mathrm{HR}<40\end{array}$ & $\mathrm{SpO}_{2}<90$ & Rojo \\
\hline $\begin{array}{l}130<\mathrm{HR}<150 \\
40<\mathrm{HR}<60\end{array}$ & $90<\mathrm{SpO}_{2}<95$ & Amarillo \\
\hline $60<\mathrm{HR}<130$ & $95<\mathrm{SpO}_{2}$ & Verde \\
\hline
\end{tabular}

se basa en cuatro niveles de prioridad.

En primer lugar, se evalúa el pulso para comprobar si éste es demasiado bajo, inferior a 10 ppm, o nulo. Si, además, la víctima presenta un nivel de $\mathrm{SpO}_{2}$ inferior al $70 \%$, adquiere una prioridad no urgente (color negro o LEDs apagados). Si la víctima presenta unos niveles de $\mathrm{SpO}_{2}$ inferiores al $90 \%$ y una frecuencia cardíaca mayor que 150 ppm (taquicardia) o menor que 40 ppm (bradicardia), presenta una prioridad muy urgente (color rojo). Si el nivel de $\mathrm{SpO}_{2}$ se encuentra entre el $95 \%$ y el $90 \%$ y la frecuencia cardíaca se encuentra entre 130 ppm y 150 ppm o 40 ppm y 60 ppm, la prioridad es urgente (color amarillo). Si el nivel de pulso es normal (entre 60 ppm y 130 ppm) y el nivel de $\mathrm{SpO}_{2}$ es superior al $95 \%$, la víctima presenta una prioridad no urgente (color verde). En la Tabla 1 se muestra de forma simplificada este algoritmo.

\section{COLOCACIÓN MEDIANTE MANIPULADOR AÉREO}

El sistema de colocación automática de los sensores para monitorización de pacientes se basa en un manipulador acoplado a un octocóptero que se controla de manera teleoperada para la búsqueda de víctimas. Cuando se encuentra una víctima en el campo visual de la cámara del manipulador, se fija la posición y el sistema detecta automáticamente las coordenadas de la muñeca de la persona. Cuando la posición objetivo se encuentra en el rango del manipulador, se inicia un movimiento rápido de colocación del sensor.

\subsection{Localización de la muñeca en las víctimas}

Se ha implementado un método basado en técnicas de visión por computador e inteligencia artificial. Este método proporciona las coordenadas cartesianas, si las hay, de la muñeca de una persona que se encuentre en el campo visual de la cámara del manipulador. 

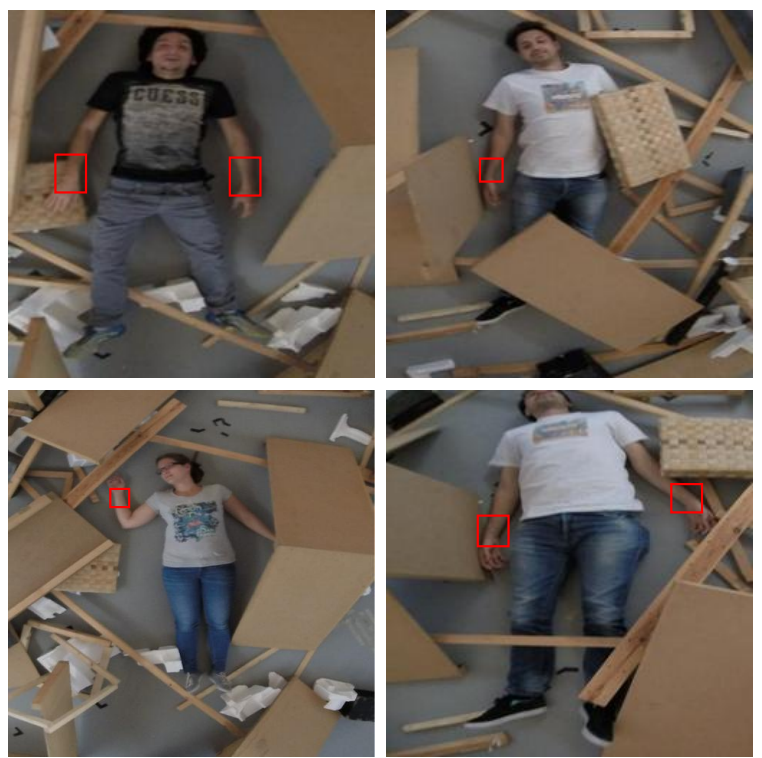

Figura 8: Cuatro imágenes del dataset utilizado para entrenamiento y validacion del detector de muñecas.

Para ello se ha utilizado una red convolucional VGG-16 [10]. Para su entrenamiento se ha creado un dataset de imágenes etiquetadas, con información de las posiciones de las muñecas de personas que aparecen en ellas. En las imágenes de la Figura 8 se representan ejemplos de las base de datos utilizadas.

El sistema de detección utiliza una red neuronal para la detección de muñecas humanas basado en imágenes de profundidad obtenidas mediante una cámara RGB-D que permiten obtener una nube de puntos mediante la Point Cloud Library (PCL) como se muestra en la Figura 9. Una vez obtenida la nube de puntos, para cada uno de ellos se evalúa la probabilidad de pertenecer a una imagen de una muñeca humana. El punto con mayor probabilidad que supera un determinado umbral es obtenido como una posible posición objetivo, cuyas coordenadas 3D en el espacio de la tarea son obtenidas a partir de los datos de la nube de puntos.

\subsection{Manipulador delta}

Por seguridad es conveniente separar el drone de la víctima. Para ello se usa un manipulador de tipo delta, con estructura de fibra de carbono. Tiene un espacio de trabajo con un radio aproximado de un metro. El manipulador tiene un peso de $2,2 \mathrm{Kg}$ y soporta una carga aproximada de $3,5 \mathrm{~kg}$. El elemento terminal puede alcanzar una velocidad máxima en la posición óptima de $4 \mathrm{~m} / \mathrm{s}$ aproximadamente. El manipulador delta consta de tres motores Dynamixel MX-64AR. Tienen un peso de 135 gramos cada uno y una velocidad máxima de $78 \mathrm{rpm}$.
En la imagen 10 se puede ver que se han construido también unas patas retráctiles de gran tamaño, de forma que se pueda realizar el despegue y aterrizaje con el manipulador acoplado al drone. Durante el despegue o aterrizaje el manipulador se contrae a una posición de reposo donde las patas puedan ejercer su función de soporte y se evite la colisión entre ambos. Una vez en el aire, las patas se repliegan, permitiendo el movimiento libre del manipulador paralelo.

El manipulador, a su vez, dispone de un elemento terminal que está en proceso de patente.

\subsection{Control de movimientos}

El manipulador parte de la posición de reposo, replegada, con el extremo situado en la posición más próxima alcanzable al centro de gravedad del conjunto.

Una vez que se tienen las coordenadas del punto de destino, se genera una trayectoria cartesiana rectilínea con la máxima velocidad, en la linea que une el centro de gravedad del manipulador aéreo y el objetivo (muñeca de la persona).

Una vez alcanzado el final de la trayectoria, o se haya producido la detección del contacto, el brazo contrae de nuevo hasta la posición de reposo.

\section{EXPERIMENTOS Y RESULTADOS}

Se han llevado a cabo experimentos para determinar la fiabilidad del sistema desarrollado. Para ello, se han recogido muestras durante 30 segundos de dos sujetos sanos, empleando simultáneamente un oxímetro de pulso comercial como referencia, aprobado para uso clínico, y el sensor MAX30102, incluido en la pulsera. En la Figura 11 se muestra el sistema experimental utilizado para comprobar el funcionamiento del sensor.

En la Figura 12 se muestran los resultados obtenidos para ambos sujetos. En el experimento realizado se puede observar que a partir de la segunda mitad del experimento los valores recogidos por el pulsioxímetro de referencia se asemejan a los valores recogidos por el MAX30102. Esto se debe a que el pulsioxímetro comercial necesita un tiempo de autocalibración antes de entregar medidas fiables. Mientras que en el sistema desarrollado con el sensor MAX30102 se ha incorporado un sistema de calibración previo al experimento. En las curvas de frecuencia cardíaca se puede apreciar que el MAX30102 presenta un error de offset que disminuye conforme la medida del sensor de referencia alcanza su valor de establecimiento. En las curvas de $\mathrm{SpO}_{2}$ se pueden apreciar algunos picos en los 


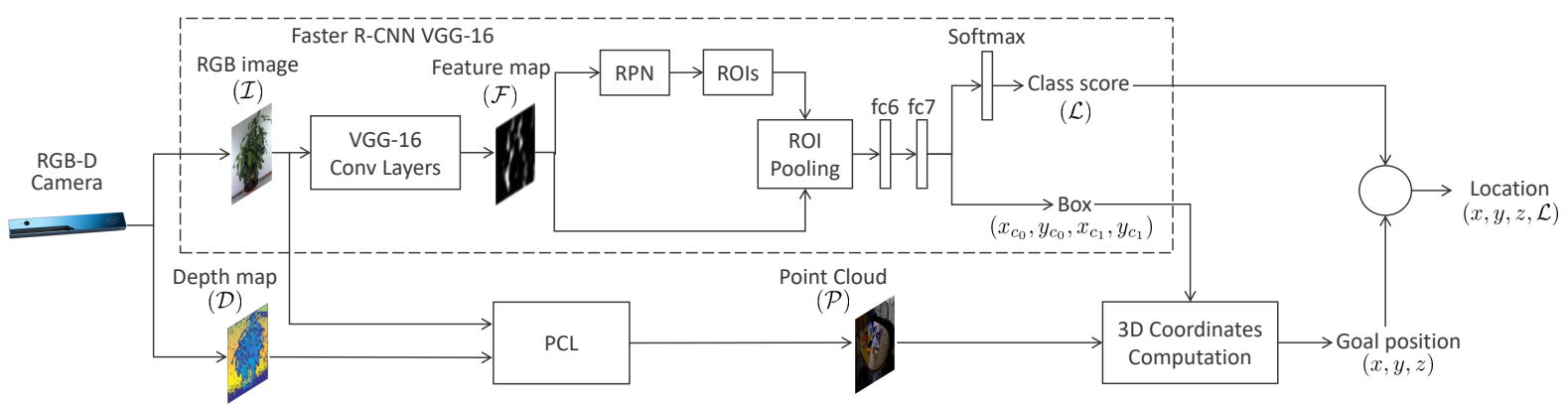

Figura 9: Sistema para la detección de muñecas humanas basado en la red neuronal Faster $R$-CNN $V G G-16$ e imágenes de profundidad RGB-D mediante la Point Cloud Library (PCL). Una vez obtenida la posición del objetivo en la imagen, se convierte a coordenadas de la escena 3D para su uso como objetivo para el manipulador aéreo.

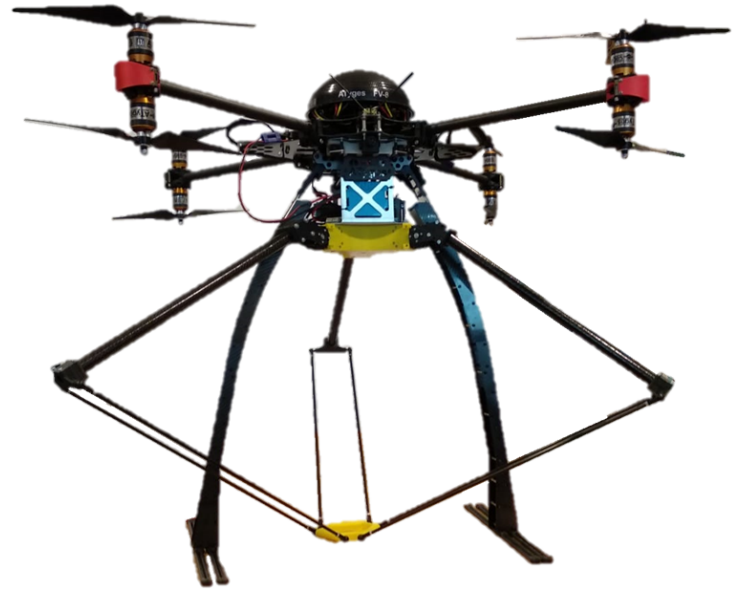

Figura 10: Fotografía del conjunto: UAV con manipulador paralelo acoplado y patas retráctiles en posición para el despegue y aterrizaje.

datos recogidos por el pulsioxímetro de referencia. Esto se debe al sistema de adquisición de datos utilizado. Para tal fin se ha empleado la shield e-health de arduino, que dispone de sus propias librerías para la toma de datos. Cabe destacar que algunos de los valores de $\mathrm{SpO}_{2}$ leídos al utilizar dichas librerías no coinciden con los mostrados en el display en el pulsioxímetro de referencia, debido a errores introducidos en la adquisición con la shield e-health. Estas diferencias se pueden apreciar en las gráficas $\mathrm{SpO}_{2}$ Sujeto 1 y $\mathrm{SpO}_{2}$ Sujeto 2 de la Figura 12 en forma de saltos o picos.

\section{CONCLUSIONES Y TRABAJOS FUTUROS}

En este trabajo se ha presentado el desarrollo de un sistema de monitorización de víctimas de forma remota. El sistema está compuesto por un UAS que es capaz de colocar sensores que miden distin- tas constantes vitales, en la muñeca de víctimas potenciales. Asimismo, se han integrado técnicas de aprendizaje profundo para la detección y el acoplamiento automático de una pulsera sensorizada a la víctima.

A la vista de los resultados obtenidos, es necesario mejorar la librería desarrollada para el cálculo de la frecuencia cardíaca. Asimismo, se pueden emplear distintos sensores de medidas de constantes vitales y comparar su comportamiento. Por otro lado, se propone mejorar el diseño para proporcionar mayor apantallamiento al sensor, disminuyendo la influencia de las condiciones de luz ambiental. También se propone el uso de tecnología LoRa para aumentar significativamente el alcance del nodo y la integración de un módulo GPS para la obtención de la localización de la víctima. Finalmente, se propone como línea de trabajo futura la incorporación de nuevos sensores y el uso de técnica de fusión sensorial para obtener una estimación del estado de salud robusta y tolerante a fallos.

Los materiales utilizados para la fabricación aditiva del prototipo poseen una baja resistencia a temperaturas (hasta $70^{\circ}$ ) y radiación $\mathrm{UV}$, por lo que deben ser sustituidos por otros que presenten mejores propiedades mecánicas como PETG o poliamida (nylon) y para su proceso de fabricación pueden usarse sinterizado que ofrece mejores tolerancias y propiedades mecánicas.

\section{Agradecimientos}

Este trabajo ha sido parcialmente financiado por el proyecto nacional DPI2015-65186-R, la Universidad de Málaga y la comisión europea en virtud del acuerdo de subvención BES-2016-078237. 


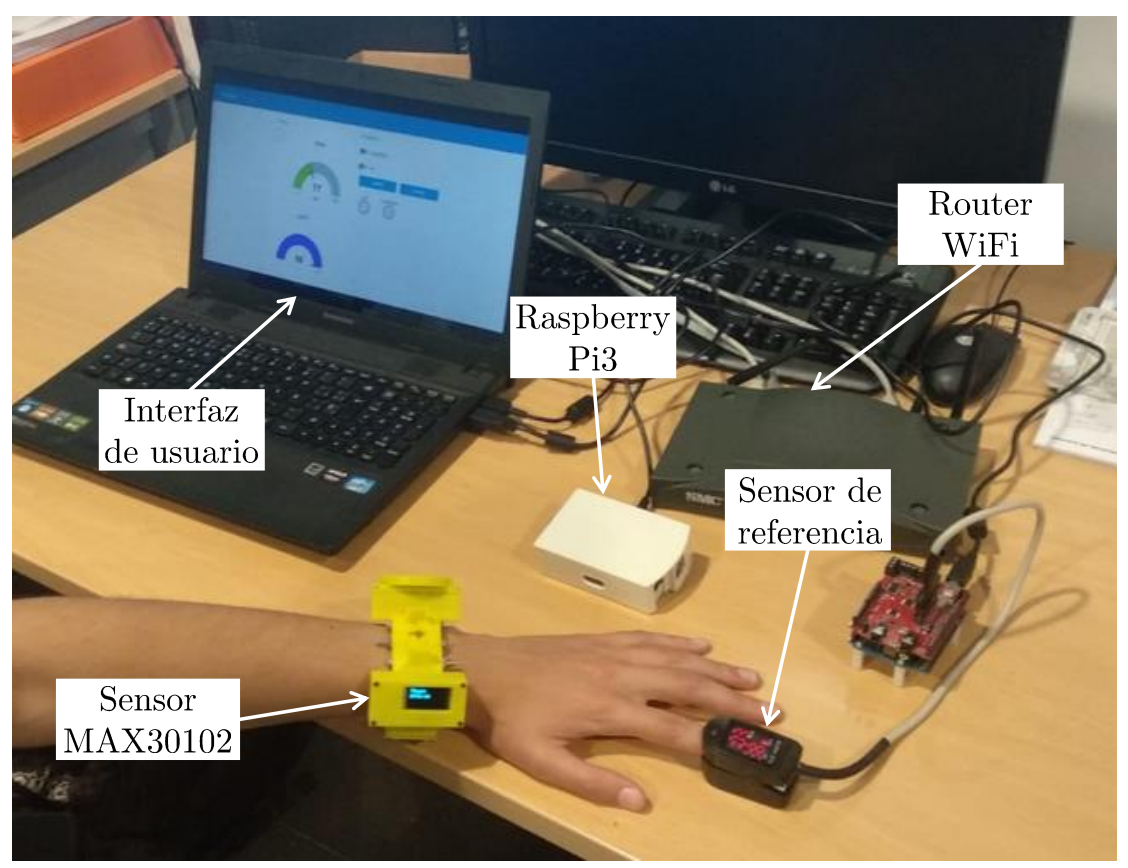

Figura 11: Sistema experimental para la validación de las medidas obtenidas con el sensor MAX30102.
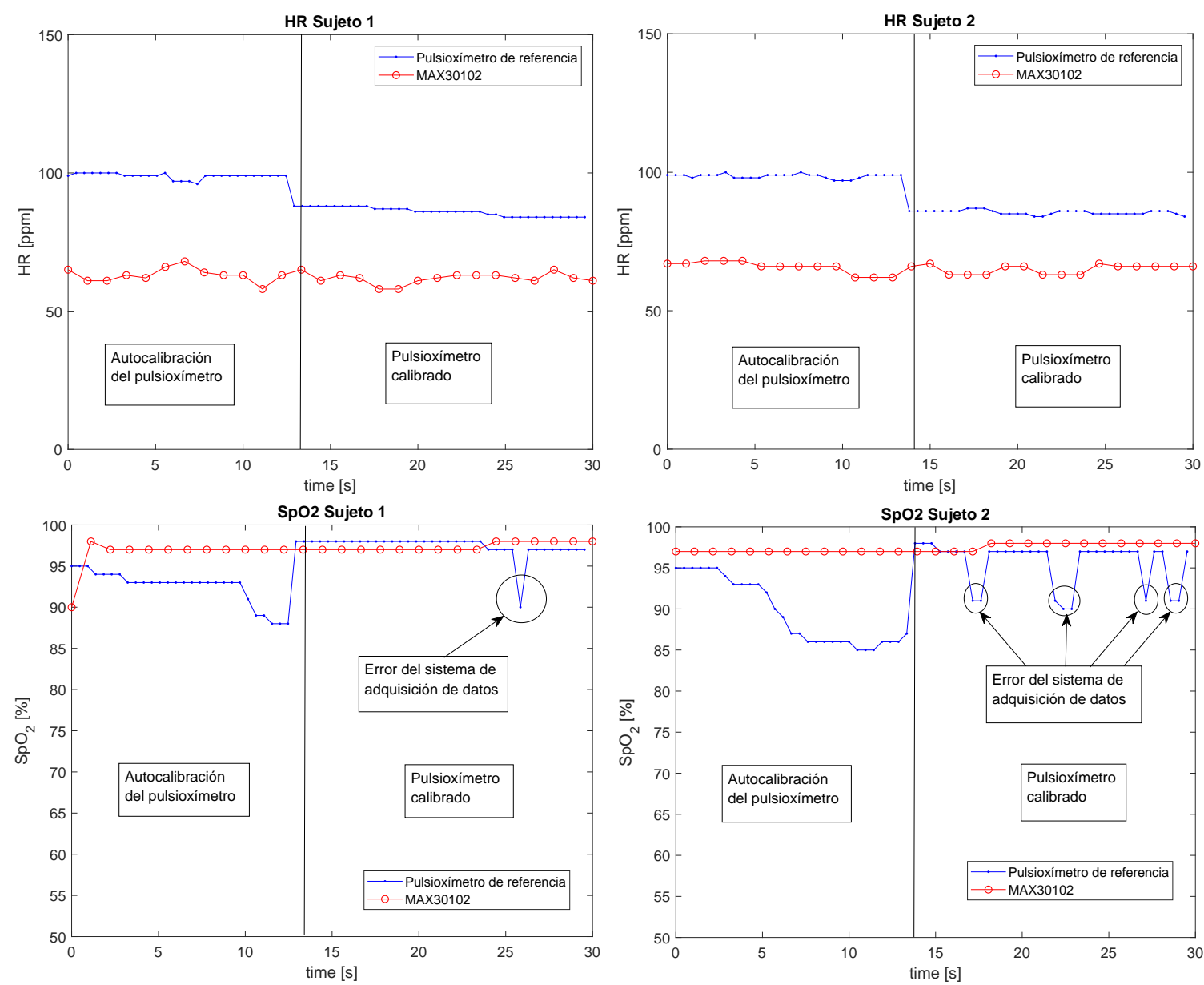

Figura 12: Representación de las muestras recogidas de frecuencia cardíaca y saturación de oxígeno en dos sujetos sanos para validar el comportamiento del sensor MAX30102. 


\section{English summary}

\section{Victims monitoring with aerial mani- pulators in search and rescue opera- tions}

\begin{abstract}
In this paper, the first device for monitoring casualties including automatic placement with aerial robotic manipulators is presented. The distributed system allows the continuous evaluation of the health status of victims in massive disaster scenarios. The design of the sensor and the communication system is described. The continuous monitoring system has many advantages against the current triage methodologies. It can obtain time series from the collected data by ehealth sensors. The sensors integrated in the wereable device provide information about vital signs that are published via Internet of Things (IoT) communication protocols for remote sensing. Furthermore, the application of attaching sensors using Unmanned Aerial Systems (UAS) is also introduced. Deep learning techniques are applied for autonomously detecting the pose of the wrist of the victim. Preliminary experiments and results show the functionality of the remote sensing system.
\end{abstract}

Keywords: Rescue robotics, Monitoring, Internet of Things, 3D printing.

\section{Referencias}

[1] Bayo-Monton, J.-L., Martinez-Millana, A., Han, W., Fernandez-Llatas, C., Sun, Y. and Traver, V.: 2018, Wearable Sensors Integrated with Internet of Things for Advancing eHealth Care, Sensors 18(6), 1851.

[2] Dhivya, A. J. A. and Premkumar, J.: 2017, Quadcopter based technology for an emergency healthcare, Third International Conference on Biosignals, Images and Instrumentation (ICBSII), pp. 1-3.

[3] Gandarias, J. M., Gómez-de Gabriel, J. M. and García-Cerezo, A. J.: 2018, Enhancing Perception with Tactile Object Recognition in Adaptive Grippers for Human-Robot Interaction, Sensors 18(3), 692.
[4] Gao, T., Hauenstein, L. K., Alm, A., Crawford, D., Sims, C. K., Husain, A. and White, D. M.: 2006, Vital signs monitoring and patient tracking over a wireless network, Johns Hopkins APL Technical Digest (Applied Physics Laboratory) 27(1), 66-73.

[5] Gómez-de-Gabriel, J. M., Gandarias, J. M., Pérez-Maldonado, F. J., García-Núñez, F. J., Fernández-García, E. J. and García-Cerezo, A. J.: 2018, Methods for autonomous wristband placement with a search-and-rescue aerial manipulator, IEEE/RSJ International Conference on Intelligent Robots and Systems (IROS).

[6] Ito, K., Sugano, S. and Iwata, H.: 2010, Development of attachable tele-echography robot by a bystander at injury scene, IEEE International Conference on Mechatronics and Automation, pp. 1270-1275.

[7] Leonardo Ristori, H.: 2011, Respuesta prehospitalaria al evento con múltiples víctimas, Revista Médica Clínica Las Condes 22(5), 556565.

[8] Lorincz, K., Malan, D., Fulford-Jones, T., Nawoj, A., Clavel, A., Shnayder, V., Mainland, G., Welsh, M. and Moulton, S.: 2004, Sensor Networks for Emergency Response: Challenges and Opportunities, IEEE Pervasive Computing 3(4), 16-23.

[9] Murphy, R. R., Srinivasan, V., Henkel, Z., Suarez, J., Minson, M., Straus, J. C., Hempstead, S., Valdez, T. and Egawa, S.: 2013, Interacting with trapped victims using robots, IEEE International Conference on Technologies for Homeland Security (HST), pp. 32-37.

[10] Simonyan, K. and Zisserman, A.: 2014, Very deep convolutional networks for largescale image recognition, arXiv preprint arXiv:1409.1556 .

[11] Ugarte, C., Tieffenberg, J. A., Amsalu, R., Romig, L. E. and Vu, T. T.: 2011, Planning and triage in the disaster scenario, American Academy of Pediatrics, Pediatric Education in Disasters Manual, Mocule 3.

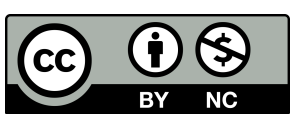

(c) 2018 by the authors. Submitted for possible open access publication under the terms and conditions of the Creative Commons Attribution CC-BY-NC 3.0 license (http://creativecommons.org/licenses/by-nc/3.0/). 